\title{
Body Falling Gesture Recognition Based on SOM and Triaxial Acceleration Information
}

\author{
Hongbo Chen ${ }^{1, *}$, Qing Gao ${ }^{1}$, Tao Feng ${ }^{1}, \mathrm{Yu} \mathrm{Liu}^{1}$ and Xinhua Xiao ${ }^{2}$ \\ ${ }^{1}$ School of Life and Environmental Science, Guilin University of Electronic Technology, Guilin 541004, China \\ ${ }^{2}$ Guangxi Kingon Software Co, Ltd, Nanning 530013, China \\ ${ }^{*}$ Corresponding author
}

\begin{abstract}
In order to improve the performance of fall detection system for the elderly based on triaxial acceleration sensor, and accurately to judge the fall direction of human body, a method was put forward based on self-organizing map neural network (SOM) and the information of triaxial acceleration sensor to cluster and analyze the human motion. To verify the recognition results of the SOM method, 130 samples of 13 common action including fall were participated in the SOM network testing. The results show that the sensitivity, specificity and accuracy of the new system were $90 \%, 96.7 \%, 94.6 \%$, respectively. These results were better than those of the method of threshold value.
\end{abstract}

Keywords-human fall gesture recognition; self-organizing map (SOM); triaxial acceleration sensor

\section{INTRODUCTION}

Problems in the health and safety monitoring of older adults have elicited research attention. The incidence of falls among older adults is extremely high. Falls can cause disability, as well as seriously affect the health and daily life of older adults, and may even result in death [1]. To reduce the harm caused by falls, a fall detection system can send an alarm to provide timely help for the victim.

At present, fall detection methods based on triaxial acceleration sensors are the mainstream methods used in fall monitoring systems for older adults [2-4]. The threshold method is a fall recognition algorithm that is commonly used in such fall detection systems [5-8]. This method can determine whether a body falls based on the changes in triaxial acceleration information, and the fall judgment threshold is a summary of the results of many experiments. However, such methods have disadvantages. On the one hand, selecting the threshold is difficult under different scenarios, which will possibly result in a relatively high false rate of the system. On the other hand, direction information after a fall, which may also be associated with several health conditions, is essential. However, the threshold method in an actual monitoring process cannot obtain the direction information of falls.

As a competitive and unsupervised learning method, a selforganizing map (SOM) neural network demonstrates powerful feature extraction capabilities and has been applied to pattern recognition [9-12]. To address the limitations of a single sensor and the deficiencies of the threshold method, a SOM neural network method was proposed to improve the recognition rate of fall monitoring systems and to gain the direction information of falls through the cluster analysis of information from a triaxial acceleration sensor.

\section{SOM Neural Network MOdEL}

A SOM neural network [13-14] can automatically and independently identify the mechanism of objects. Through a SOM, the same objects will cluster in the same region of a feature space, whereas different classes of objects will cluster in different regions of a feature space.

A typical SOM network structure (Figure I) comprises an input layer and a competitive layer. The input layer consists of a number of $m$ neurons, whereas the competitive layer is a $2 \mathrm{D}$ array with a number of $\mathrm{a} \times \mathrm{b}$ neurons. The neurons in the input layer and the competitive layer demonstrate full connectivity with each other, and every neuron in the competitive layer exhibits lateral inhibition connection with one another. First, the sample data, which are the input from the input layer, will be weighted using the connection weights between the two layers. Then, a set of output values will be obtained in the output layer.

The steps for training a SOM neural network are as follows.

(1) Network initialization. The initial values of the weights between the input layer and the competitive layer are set using random numbers.

(2) Winning neuron calculation. A random training sample is drawn and then the winning neuron is calculated.

(3) Weight updating. The weights of the winning neuron and its neighborhood are updated.

(4) Learning rate and neighborhood updating. The learning rate and the neighborhood are updated before the next iteration.

(5) Iteration end judgment. If the sample does not finish learning, then another training sample is randomly selected; hence, step (2) is repeated. Otherwise, the iteration is terminated.

Each node in the competitive layer is mutually stimulated to learn; thus, nodes that are close to one another after training share similar weights. The spatial positions of the output nodes of a SOM network reflect the internal relations of the input samples; that is, inputs with similar properties will be mapped onto nearby SOM output nodes. 


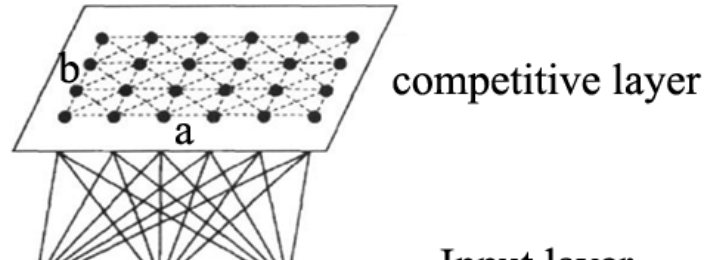

Input layer

FIGURE I. STRUCTURE OF A TYPICAL SOM NEURAL NETWORK

\section{DATA COLLECTION}

We establish a dynamic 3D Cartesian coordinate system $\mathrm{O}$-xyz on a standing upright human body with a triaxial acceleration sensor placed on the waist, as shown in Figure II. The acceleration components of the $\mathrm{x}-, \mathrm{y}-$, and $\mathrm{z}$-axes are denoted as $\mathrm{a}_{\mathrm{x}}, \mathrm{a}_{\mathrm{y}}$, and $\mathrm{a}_{\mathrm{z}}$, respectively.

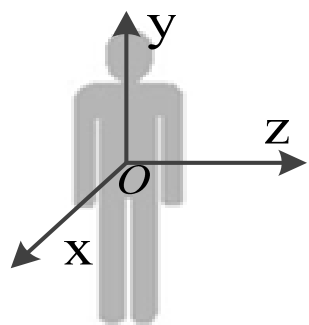

FIGURE II. WAIST TRIAXIAL ACCELERATION SENSOR AND THE DYNAMIC 3D CARTESIAN COORDINATE OF THE BODY

Five young participants with good physical fitness are invited to simulate the fall and non-fall actions of older adults. Fall actions include falling forward, falling backward, falling to the left, and falling to the right. Non-fall actions are normal daily life actions, including nine typical actions, namely, moving upstairs, moving downstairs, walking on flat ground, standing up, sitting down, before bending, jumping, running, and squatting.

In the fall detection experiments, we have collected information from the waist triaxial acceleration sensor for the 13 types of actions. A total of 20 samples are collected for each type of action, and 260 feature samples of the 13 types of actions are obtained. Among the feature samples, 10 are randomly selected as the training set, whereas the remaining samples are used as the test set. Thus, a total of 130 samples are included in the training and test sets.

\section{Constructing the Feature Space}

We have adopted the sliding window method to extract sample data. The sliding window size is set to $3 \mathrm{~s}$, whereas the sampling frequency is set to $15 \mathrm{~Hz}$. The feature points of each sample can reach 45 for every channel of the sensor.
In the fall detection experiments using the waist acceleration sensor, each sample includes information on triaxial acceleration. Thus, the sample is a 135-dimensional $(45 \times 3)$ feature space.

\section{Results OF THE FALl DETECTION EXPERIMENTS AND Clustering ANALYSIS}

\section{A. Clustering Results}

The training set includes 130 samples, and training time is set to 1000 . The competitive layer of the proposed SOM network adopts the output structures of a $2 \mathrm{D}$ rectangular planar array that measures $14 \times 13$. Figure III presents the clustering results of the fall detection experiments based on the triaxial

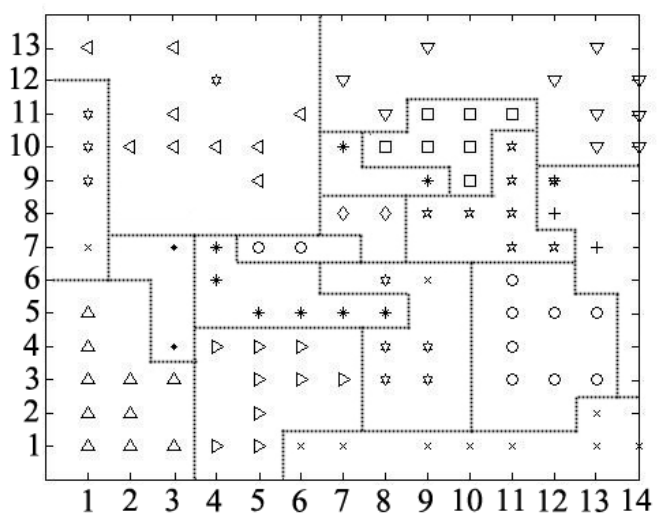

(a)

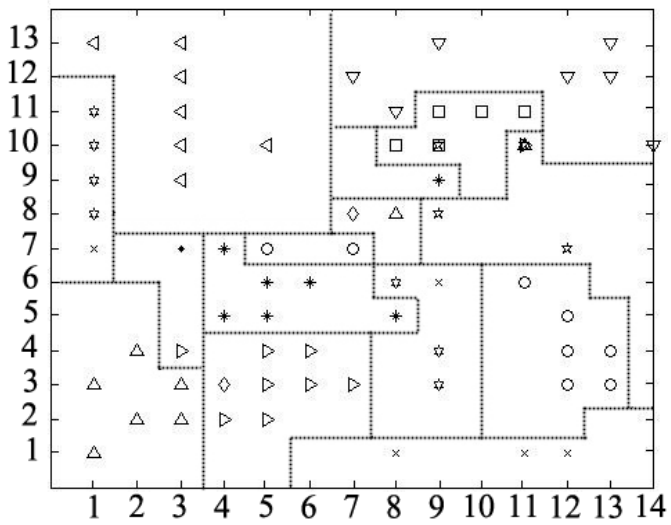

(b)

FIGURE III. CLUSTERING RESULTS OF FALL DETECTION

(a) training results based on accelerometer data, (b) test results based on accelerometer data. $\Delta$ denotes falling forward, $\nabla$ denotes falling backward, $\triangleleft$ denotes falling to the left, $\triangleright$ denotes falling to the right, $*$ denotes moving upstairs, $O$ denotes moving downstairs, $\square$ denotes walking on flat ground, $\diamond$ denotes standing up, it denotes sitting down, $\bullet$ denotes before bending, $\times$ denotes jumping, a hexagon denotes running, and + denotes squatting. Various graphics may overlap with one another, which indicates that some types of action samples 
cluster in the neurons. The dotted line is the dividing line of the 13 types of action samples in the classification results.

Accelerometer information. Figures III (a) and III (b) indicate that 13 types of actions can be successfully recognized using single sensor information for the cluster analysis; in particular, four types of fall and non-fall actions can be effectively separated. However, erroneous identification cases occur, such as those shown in Figure III (b); that is, falls to the right side are identified as bending over, sitting down, and other non-fall actions, which results in missed detection of fall actions.

\section{B. Analysis and Evaluation of the Results}

To further illustrate the effectiveness of the SOM method, we compare it with the conventional threshold method.

We adopt three performance indicators, namely, sensitivity, specificity, and accuracy, to evaluate the fall detection results [15-16]. Sensitivity (Se) is defined as the detection rate of fall actions.

$$
\mathrm{Se}=\frac{T P}{T P+F N}
$$

TABLE I. RECOGNITION RESULTS OF THE SOM METHOD

\begin{tabular}{|c|c|c|c|c|c|c|c|c|c|c|c|c|c|}
\hline & $\Delta$ & $\nabla$ & $\triangleleft$ & $\triangleright$ & * & $\circ$ & 口 & $\diamond$ & $\hat{i}$ & $\bullet$ & $x$ & $\Rightarrow$ & + \\
\hline$\Delta$ & 8 & & & & & & & & & & & & \\
\hline$\nabla$ & & 10 & & & & & & & & & & & \\
\hline$\triangleleft$ & & & 10 & & & & & & & & & & \\
\hline$\triangleright$ & & & & 8 & & & & 3 & & & & & \\
\hline$*$ & & & & & 10 & & & & & & & & \\
\hline 0 & & & & & & 10 & & & & & & & \\
\hline$\square$ & & & & & & & 10 & & 5 & & & & \\
\hline$\nabla$ & 1 & & & & & & & 7 & & & & & \\
\hline $\bar{i}$ & 1 & & & 1 & & & & & 5 & & & & \\
\hline • & & & & 1 & & & & & & 10 & & & \\
\hline$x$ & & & & & & & & & & & 10 & & \\
\hline$\Rightarrow$ & & & & & & & & & & & & 10 & \\
\hline+ & & & & & & & & & & & & & 10 \\
\hline$\%$ & 80 & 100 & 100 & 80 & 100 & 100 & 100 & 70 & 50 & 100 & 100 & 100 & 100 \\
\hline
\end{tabular}

$\Delta$ denotes falling forward, $\nabla$ denotes falling backward, $\triangleleft$ denotes falling to the left, $\triangleright$ denotes falling to the right, * denotes moving upstairs, $\bigcirc$ denotes moving downstairs, $\square$ denotes walking on flat ground, $\Delta$ denotes standing up, $i>$ denotes sitting down, $\bullet$ denotes before bending, $\times$ denotes jumping, $\rightarrow$ denotes running, and + denotes squatting. Various graphics may overlap with one another, which indicates that some types of action samples cluster in the neurons. The dotted line is the dividing line of the 13 types of action samples in the classification results.

Specificity $(\mathrm{Sp})$ is the detection rate of normal actions.

$$
\mathrm{Sp}=\frac{T N}{T N+F P}
$$

Accuracy (Ac) is the detection rate of all the actions.

$$
\mathrm{A} c=\frac{T P+T N}{T P+F N+T N+F P}
$$

In the preceding formulas, TP (true positive) indicates the number of fall samples that are detected as fall actions, FP (false positive) indicates the number of non-fall samples that are detected as fall actions, TN (true negative) indicates the number of non-fall samples that are detected as non-fall actions, and FN (false negative) indicates the number of fall samples that are detected as non-fall actions.

Table I shows the results of the proposed method and Table II shows results comparation between the SOM method and the threshold method. Regardless of the information from the triaxial acceleration sensor, the sensitivity, specificity, and accuracy of the cluster analysis method of the proposed SOM are higher by approximately $10 \%$ than those of the threshold method.

TABLE II. RECOGNITION RESULTS OF THE SOM METHOD AND THE THRESHOLD METHOD

\begin{tabular}{ccc}
\hline & SOM & Threshold \\
\hline Total number of samples & 130 & 130 \\
Fall action samples & 40 & 40 \\
Non-fall action samples & 90 & 90 \\
True positive samples & 36 & 32 \\
False positive samples & 3 & 14 \\
True negative samples & 87 & 76 \\
False negative samples & 4 & 8 \\
Sensitivity (\%) & $90.0 \%$ & $80.0 \%$ \\
Specificity (\%) & $96.7 \%$ & $84.4 \%$ \\
Accuracy (\%) & $94.6 \%$ & $83.1 \%$ \\
\hline
\end{tabular}

\section{CONCLUSIONS}

In this study, the cluster analysis of human actions have been performed via a novel SOM neural network using information fusion obtained from a waist triaxial acceleration sensor. The method has recognized human fall gestures, and the following conclusions are drawn.

1) Through the proposed SOM neural network, the cluster analysis of the waist triaxial acceleration sensor information can effectively identify the directions of falls, whereas the conventional threshold method cannot recognize human fall direction. 
2) For recognizing human falls, the sensitivity, specificity, and accuracy of the cluster analysis method of the SOM neural network are higher than those of the conventional threshold method; thus, the SOM exhibits better recognition effect.

3) The SOM algorithm for recognizing human falls demonstrates higher reliability and stronger suitability than the conventional threshold method.

\section{ACKNOWLEDGMENTS}

We would like to express our gratitude to the individuals who participated in this study and generously contributed their time and effort. This research was supported in part by a project grant from the National Natural Science Foundation of China to HC (81460273), a project grant from the Guangxi Natural Science Foundation to HC (2013GXNSFAA019325), and a project grant from the Guangxi Science and Technology Program to HC (1348020-10).

\section{REFERENCE}

[1] Liu-Ambrose T, Davis J, Chun L. H. et al., secondary falls prevention in community-dwelling senior fallers: study protocol for a randomized controlled trial, Trials, 16(2015) 1-9.

[2] Sriborrirux, W.; Leamsumran, P.; Dan-klang,P.. Real-time system for monitoring activity among the elderly using an RF $\mathrm{SoC}$ device with triaxial accelerometer data over a wireless sensor network:2014 IEEE MTT-S International Microwave Workshop Series on RF and Wireless Technologies for Biomedical and Healthcare Applications(IMWSBio),Thailand,December 8-10,2014 [C].IEEE,2014.

[3] Yanjun Li,Gan Chen, Yueyun Shen,et al. Accelerometer- based fall detection sensor system for the elderly:2012 IEEE 2nd International Conference on Cloud Computer and Intelligent Systems (CCIS),Hangzhou, China, October 30- November 1,2012[C].IEEE,2012.

[4] Aguiar,B.;Rocha,T.;Silva,J.; et al. Accelerometer- based fall detection for smartphones: 2014 IEEE International Symposium on Medical Measurements and Applications (MeMeA), June 1112, 2014 [C].IEEE,2014.

[5] Gibson R M, Amira A, Ramzan N, et al., Multiple comparator classifier framework for accelerometer-based fall detection and diagnostic, Applied Soft Computing, 39(2016)94-103.

[6] Kwolek B, and Kepski M, Improving fall detection by the use of depth sensor and accelerometer, Neurocomputing, 168(2015) 637- 645.

[7] Habaebi M H, AGEL M M, and ZYOUD A, Performance of ZigBee Based Fall Detection Alarm System, IEICE TRANSACTIONS on Communications, E99-B(2016)385-391.

[8] Leone A, Rescio G, Caroppo A, et al., A Wearable EMG-based System Pre-fall Detector, Procedia Engineering, 120(2015)455458.

[9] Sun Q, Liu H, Liu M, et al., Human activity prediction by mapping grouplets to recurrent Self-Organizing Map, Neurocomputing, 177(2016) 427-440.

[10] Far A B, Flitti F, Guo B et al., A Bio-Inspired Pattern Recognition System for Tin-Oxide Gas Sensor Applications, IEEE SENSORS JOURNAL, 9(2009)713-722.

[11] Harada Y, Tomoki K, Kanzaki R et al., Response prediction of an insect's olfactory receptor neuron by using structural arameters of odorant and Self-Organizing Map, IEEE Sensors Journal, 14(2014)1014 - 1017.

[12] Chea R, Grenouillet G, and Lek S, Evidence of Water Quality Degradation in Lower Mekong Basin Revealed by SelfOrganizing Map, PLoS ONE, 11(2016)1-19.
[13] Kuremoto,T.;Kobayashi,K.;Otani,T.;et al. One dimensional ring type growing SOM with asymmetric neighborhood function and its application to a hand shape instruction learning system:2014 $15^{\text {th }}$ IEEE/ACIS International Conference on Software Engineering,Artificial Intelligence,Networking and Parallel/Distributed Computing(SNPD), June 30-July 2, 2014[C] IEEE, 2014.

[14] Vojacek,L.;Dvorsky,J.;Slaninova,K.; et al. Scalable parallel SOM learning for web user profiles:2013 $13^{\text {th }}$ International Conference on Intelligent Systems Design and Applications(ISDA), December 8-10,2013[C].IEEE,2013.

[15] Bolboacă S D, and Jäntschi L, Sensitivity, specificity, and accuracy of predictive models on phenols toxicity, Journal of Computer Science, 5(2014)345-350.

[16] McShefferty D, Whitmer W M, Swan I R C et al., The effect of experience on the sensitivity and specificity of the whispered voice test: a diagnostic accuracy study, BMJ Open, 3(2013)e002394. 